\title{
Article
}

\section{In Vitro Activity and Clinical Outcomes of Clofazimine for Nontuberculous Mycobacteria Pulmonary Disease}

\author{
Dae Hun Kim ${ }^{1, \dagger}{ }^{\text {, Bo-Guen Kim }}{ }^{1,+} \oplus$, Su-Young Kim ${ }^{1}$, Hee Jae Huh ${ }^{2} \oplus$, Nam Yong Lee ${ }^{2}$, Won-Jung Koh ${ }^{1, \ddagger}$, \\ Hojoong Kim ${ }^{1}{ }^{1}$, O Jung Kwon ${ }^{1}$ and Byung Woo Jhun ${ }^{1, *}$ \\ 1 Samsung Medical Center, Department of Medicine, Division of Pulmonary and Critical Care Medicine, \\ Sungkyunkwan University School of Medicine, Seoul 06351, Korea; tnxo515@gmail.com (D.H.K.); \\ kbg1q2w3e@gmail.com (B.-G.K.); suyoung5505@gmail.com (S.-Y.K.); healthylung@gmail.com (W.-J.K.); \\ hjk3425@skku.edu (H.K.); ojkwon@skku.edu (O.J.K.) \\ 2 Samsung Medical Center, Department of Laboratory Medicine and Genetics, Sungkyunkwan University \\ School of Medicine, Seoul 06351, Korea; pmhhj77@gmail.com (H.J.H.); micro.lee@samsung.com (N.Y.L.) \\ * Correspondence: byungwoo.jhun@gmail.com \\ + These authors contributed equally to this work. \\ $\ddagger$ Deceased.
}

Citation: Kim, D.H.; Kim, B.-G.; Kim, S.-Y.; Huh, H.J.; Lee, N.Y.; Koh, W.-J.; Kim, H.; Kwon, O.J.; Jhun, B.W. In Vitro Activity and Clinical Outcomes of Clofazimine for Nontuberculous Mycobacteria Pulmonary Disease. J. Clin. Med. 2021, 10, 4581. https:// doi.org/10.3390/jcm10194581

Academic Editor: Luis Garcia-Marcos

Received: 21 August 2021

Accepted: 1 October 2021

Published: 3 October 2021

Publisher's Note: MDPI stays neutral with regard to jurisdictional claims in published maps and institutional affiliations.

Copyright: (c) 2021 by the authors. Licensee MDPI, Basel, Switzerland. This article is an open access article distributed under the terms and conditions of the Creative Commons Attribution (CC BY) license (https:// creativecommons.org/licenses/by/ $4.0 /)$.

\begin{abstract}
Limited data are available regarding the in vitro activity of clofazimine against nontuberculous mycobacteria (NTM) or on outcomes of clofazimine-containing regimens in NTM-pulmonary disease (PD). Therefore, we evaluated the in vitro activity of clofazimine and the clinical outcomes of clofazimine-containing regimens. We evaluated clofazimine in vitro activity for 303 NTM isolates from NTM-PD patients. Fifty-seven clarithromycin-resistant and 35 amikacin-resistant isolates were also analyzed. Culture conversion after a 12-month treatment regimen containing clofazimine was evaluated in 58 NTM-PD patients, including 20 patients with drug-resistant isolates. Most of the 303 isolates (238/303) had minimum inhibitory concentrations (MICs) $\leq 0.25 \mu \mathrm{g} / \mathrm{mL}$ for clofazimine (57/63 Mycobacterium avium, 53/57 M. intracellulare, 49/52 M. kansasii, 22/64 M. abscessus, and 57/67 M. massiliense). For the 57 clarithromycin-resistant and 35 amikacin-resistant isolates, most had MICs $\leq 0.25 \mu \mathrm{g} / \mathrm{mL}$ (47/57 and 32/35, respectively). Among the 38 NTM-PD patients without resistance to clarithromycin or amikacin, $47 \%$ achieved culture conversion (8/27 M. abscessus, 9/9 M. massiliense, 0/1 M. avium, and 1/1 M. intracellulare). The conversion rate was higher in the $\mathrm{MIC} \leq 0.25 \mu \mathrm{g} / \mathrm{mL}$ group than in the MIC $=0.5 \mu \mathrm{g} / \mathrm{mL}$ group $(13 / 18 \mathrm{vs} .5 / 20, p=0.004)$, and an MIC $\leq 0.25 \mu \mathrm{g} / \mathrm{mL}$ remained a significant factor in multivariable analysis. Culture conversion was achieved in $20 \%$ of 20 patients with clarithromycin- or amikacin-resistant isolates. However, a clofazimine MIC $\leq 0.25 \mu \mathrm{g} / \mathrm{mL}$ was not significant for culture conversion in the 58 NTM-PD patients, regardless of the drug resistance pattern. Clofazimine was effective in vitro against NTM species. Some patients on clofazimine-containing regimens achieved culture conversion.
\end{abstract}

Keywords: nontuberculous mycobacteria; clofazimine; in vitro; minimum inhibitory concentration; minimum bactericidal concentration

\section{Introduction}

The burdens of pulmonary disease (PD) due to nontuberculous mycobacteria (NTM) are increasing globally [1,2]. Mycobacterium avium complex (MAC), mainly composed of M. avium and M. intracellulare, is the most common pathogen associated with NTM-PD, and M. abscessus, predominantly composed of M. abscessus subsp. abscessus (hereafter, M. abscessus) and M. abscessus subsp. massiliense (hereafter, M. massiliense), is the second most common pathogen in many countries. M. kansasii also causes NTM-PD [3,4].

For MAC-PD or M. kansasii-PD, guidelines recommend a macrolide-based multidrug therapy, including ethambutol and rifamycin with or without an injectable aminoglycoside $[4,5]$. However, treatment outcomes are unsatisfactory, and many patients remain 
culture-positive with refractory NTM-PD [6,7]. For M. abscessus- or M. massiliense-PD, a major challenge is that the pathogens are highly drug-resistant, despite guidelines recommending multidrug therapy, including intravenous amikacin with imipenem (or cefoxitin), based on the results of drug susceptibility testing (DST) [8]. Thus, there are only a few effective oral drugs for long-term maintenance therapy of NTM-PD in real-world practice, and improved therapeutic options are needed.

There is growing evidence on the efficacy of clofazimine for treatment of primary or refractory NTM-PD [9-13]. Clofazimine is a fat-soluble riminophenazine-based antibiotic primarily used to treat leprosy due to its lipophilicity in the skin and anti-inflammatory properties [14], and it has been used to treat drug-resistant tuberculosis [13,15,16]. Clofazimine has several advantages for NTM-PD treatment, including its long half-life, oral availability, slow metabolic elimination, ability to achieve high concentration in macrophages, and rapid localization within phagocytes [17]. Additionally, data have demonstrated synergistic effects with amikacin against NTM [18].

However, limited research exists on the in vitro antibiotic susceptibility for clinical NTM isolates. Therefore, we evaluated the in vitro activity of clofazimine against NTM, including macrolide- or amikacin-resistant clinical isolates, and clinical outcomes of NTMPD patients treated with clofazimine-containing regimens.

\section{Materials and Methods}

\subsection{Study Participants}

We evaluated 303 clinical isolates of major pathogenic NTMs obtained from 303 patients with treatment-naive NTM-PD between January 2009 and November 2018 at Samsung Medical Center, a referral hospital in Seoul, Korea. Additionally, 57 clarithromycin-resistant and 35 amikacin-resistant non-duplicated NTM isolates, all confirmed to have mutations associated with acquired resistance ( $r r l$ for clarithromycin and $r r s$ for amikacin), were included in the analysis. In vitro activity of clofazimine against these isolates was evaluated. The clinical outcome was also evaluated for 58 patients treated with clofazimine-containing regimens for $\geq 6$ months. All patients met the diagnostic criteria of the American Thoracic Society/Infectious Diseases Society of America guidelines [4]. All NTM isolates and the clinical data included in this study were obtained from an Institutional Review Boardapproved observational cohort at Samsung Medical Center (ClinicalTrials.gov identifier: NCT00970801).

\subsection{NTM Identification}

Acid-fast bacilli smears and cultures of the respiratory specimens were obtained using standard methods. The processed specimens were inoculated into the BACTEC MGIT system (BD Diagnostics, Sparks, MD, USA). Liquid cultures were used for NTM species identification and DST. Species were identified using polymerase chain reaction (PCR)restriction fragment length polymorphism analysis or reverse-blot hybridization of the $r p o B$ gene in routine clinical practice. Beginning in June 2014, species identification was conducted via nested multiplex PCR and a reverse-hybridization assay of the internal transcribed spacer (ITS) region (AdvanSureTM Mycobacteria GenoBlot Assay; LG Life Sciences, Seoul, Korea).

For research, NTM isolates from broth medium were subcultured on 3\% Ogawa agar before harvesting and storage at $-80^{\circ} \mathrm{C}$. Clinical isolates were then propagated on Middlebrook 7H10 agar plates (Difco Laboratories, Detroit, MI, USA) supplemented with $10 \%(v / v)$ oleic acid-albumin-dextrose-catalase (OADC) (BD Diagnostics). Single colonies were obtained and re-identified using multilocus sequence analysis of $h s p 65$, the 16S-23S rRNA gene ITS, erm(41), and rpoB and were retested for clofazimine susceptibility.

\subsection{In Vitro Activity against Clofazimine}

In vitro susceptibility testing of various antibiotics was performed using the broth microdilution method according to the Clinical and Laboratory Standards Institute (CLSI) 
guidelines [19,20]. Rapidly growing mycobacteria (RGM), including M. abscessus and M. massiliense, used cation-adjusted Mueller-Hinton broth (CAMHB) (Difco Laboratories), and slowly growing mycobacteria (SGM), including MAC and M. kansasii, used CAMHB with 5\% OADC according to CLSI guidelines.

Clofazimine dissolved in dimethyl sulfoxide was dispensed into CAMHB or CAMHB with 5\% OADC using serial dilution, and then the antibiotic-containing medium was dispensed into 96-well plates. A single colony of bacteria was placed in a $5 \mathrm{~mL}$ tube containing phosphate-buffered saline, and the density was adjusted to 0.5 McFarland standard. The bacterial suspension was diluted $1 / 200$, inoculated into CAMHB or CAMHB with 5\% OADC, and then dispensed into the 96-well plates.

Minimum inhibitory concentrations (MICs) were determined after incubation of RGM (3-5 days) and SGM (>10 days) at a constant temperature $\left(36^{\circ} \mathrm{C}\right)$ and humidity (75-80\%). After MIC measurement, inoculum suspensions (20-50 $\mu \mathrm{L})$ in 96-well plates at concentrations above MIC were inoculated on Middlebrook 7H10 agar with 10\% OADC without antibiotics followed by re-incubation (RGM, 3-4 days; SGM, $\geq 10$ days). Minimum bactericidal concentrations (MBCs) were determined as the lowest antibiotic concentration that yielded no visible colonies.

\subsection{Sequence Analysis for Acquired Clarithromycin and Amikacin Resistance Mutations}

Isolates were categorised as clarithromycin or amikacin resistant, respectively, if they contained any of the following $r r l$ or $r r s$ mutations associated with acquired resistance [21]: A2058G, A2058C, A2058T, A2059T, A2059C, or A2059G (Escherichia coli 23S rRNA gene position numbering) in the clarithromycin acquired resistance region of $r r l$; or A1408G, T1406A, C1409T, or G1491T in the amikacin acquired resistance region of rrs (E. coli 16S rRNA gene position numbering). To confirm rrl and rrs gene mutations, NTM DNA was extracted using the DNeasy UltraClean Microbial Kit (Qiagen, Hilden, Germany), and PCR was performed using $r r l$ ( $r r l-F ; 5^{\prime}$-TTTAAGCCCCAGTAAACGGC$3^{\prime}$; $r r l-\mathrm{R}, 5^{\prime}$-GTCCAGGTTGAGGGAACCTT-3') or $r r s$ (rrs1-F; 5'-ATGACGTCAAGTCAT CATGCC-3'; rrs1-R, 5'-AGGTGATCCAGCCGCACCTTC-3') specific primers, respectively. The following PCR conditions were used for the $r r l$ and $r r s$ regions: denaturation at $94{ }^{\circ} \mathrm{C}$ ( $2 \mathrm{~min}$ ) followed by 35 cycles $\left(94^{\circ} \mathrm{C}, 30 \mathrm{~s} ; 58.5^{\circ} \mathrm{C}, 30 \mathrm{~s} ; 72{ }^{\circ} \mathrm{C}, 3 \mathrm{~min}\right)$ and a final extension for $5 \mathrm{~min}$. The PCR products were purified and sequenced using the $r r l-\mathrm{F}$ and $r r s 1-\mathrm{F}$ primers, respectively.

\subsection{Treatment and Clinical Outcomes}

We evaluated treatment outcomes for 58 patients with NTM-PD (M. abscessus, M. massiliense, or MAC) who were treated with clofazimine-containing regimens for $\geq 6$ months. In M. abscessus- or M. massiliense-PD patients, an initial two- to four-week course of intravenous amikacin and imipenem (or cefoxitin) with or without tigecycline was administered, together with oral macrolide or clofazimine (100 mg/day), after which the oral regimen was maintained. In MAC-PD patients, a macrolide-based regimen including ethambutol and rifampin, with or without intravenous amikacin, was used. Non-liposomal inhaled amikacin was used for patients with refractory NTM-PD (Supplementary Table S1). At our institution, clofazimine was included in the initial M. abscessus or M. massiliense treatment, while clofazimine was usually added as salvage treatment in refractory MAC-PD.

Treatment outcome was based on culture conversion at 12 months after antibiotic treatment initiation. 'Culture conversion' was defined as at least three consecutive negative cultures after treatment, collected at least four weeks apart. Time of conversion was defined as the date of the first negative culture. There are two reasons for evaluating the outcomes at 12 months after treatment initiation: first, treatment outcomes could be underestimated in patients with shorter treatment periods and overestimated in those with longer treatment periods; second, in real-world clinical practice, treatment outcome is evaluated after 12 months of therapy. 


\subsection{Statistical Analysis}

Data were presented as numbers (percentages) or medians (interquartile ranges). Data were compared by the Mann-Whitney $U$ test for continuous variables and the chi-square test or Fisher's exact test for categorical variables. Multivariable analyses with a logistic regression model were performed to evaluate factors associated with culture conversion. Differences were considered statistically significant at $p<0.05$. All statistical analyses were performed using SPSS (IBM SPSS Statistics ver. 25, Chicago, IL, USA).

\section{Results}

\subsection{In Vitro Activity of Clofazimine in NTM without Drug Resistance}

Table 1 shows the MIC and MBC values of clofazimine against 303 clinical NTM isolates without resistance to clarithromycin and amikacin. Overall, the MICs of clofazimine tended to be low in all NTM species. Most of the isolates, 79\% (238/303), had clofazimine MICs $\leq 0.25 \mu \mathrm{g} / \mathrm{mL}$, including $90 \%$ (57/63) of M. avium, 93\% (53/57) of M. intracellulare, $94 \%$ (49/52) of M. kansasii, 34\% (22/64) of M. abscessus, and 85\% (57/67) of M. massiliense.

Table 1. $\mathrm{MIC}_{50}, \mathrm{MIC}_{90}, \mathrm{MBC}_{50}$, and $\mathrm{MBC}_{90}$ values of clofazimine for 303 clinical NTM isolates.

\begin{tabular}{|c|c|c|c|c|c|c|c|c|c|c|c|c|}
\hline \multirow{2}{*}{$\begin{array}{c}\text { NTM } \\
\text { Species (No.) }\end{array}$} & \multirow{2}{*}{ Activity } & \multicolumn{7}{|c|}{ No. $(\%)$ of Isolates According to MIC $(\mu \mathrm{g} / \mathrm{mL}) *$} & \multirow{2}{*}{$\begin{array}{c}\mathrm{MIC}_{50} \\
(\mu \mathrm{g} / \mathrm{mL})\end{array}$} & \multirow{2}{*}{$\begin{array}{c}\mathrm{MIC}_{90} \\
(\mu \mathrm{g} / \mathrm{mL})\end{array}$} & \multirow{2}{*}{$\begin{array}{l}\mathrm{MBC}_{50} \\
(\mu \mathrm{g} / \mathrm{mL})\end{array}$} & \multirow{2}{*}{$\begin{array}{l}\mathrm{MBC}_{90} \\
(\mu \mathrm{g} / \mathrm{mL})\end{array}$} \\
\hline & & $\leq 0.062$ & 0.125 & 0.25 & 0.5 & 1 & 2 & 4 & & & & \\
\hline \multirow{2}{*}{ M. avium (63) } & MIC & $23(36)$ & $16(25)$ & $18(29)$ & $3(5)$ & $1(2)$ & $2(3)$ & & 0.125 & 0.25 & \multirow[b]{2}{*}{0.125} & \multirow[b]{2}{*}{0.5} \\
\hline & MBC & $20(31)$ & $15(24)$ & $20(32)$ & $5(8)$ & $1(2)$ & $2(3)$ & & & & & \\
\hline \multirow[b]{2}{*}{ M. intracellulare (57) } & MIC & $5(8)$ & $20(35)$ & $28(49)$ & $2(4)$ & & $2(4)$ & & 0.25 & 0.25 & \multirow[b]{2}{*}{0.25} & \multirow[b]{2}{*}{0.25} \\
\hline & MBC & $5(8)$ & $15(27)$ & $32(56)$ & $3(5)$ & & $2(4)$ & & & & & \\
\hline \multirow{2}{*}{ M. kansasii (52) } & MIC & $45(86)$ & $3(6)$ & $1(2)$ & $1(2)$ & & $2(4)$ & & $\leq 0.062$ & 0.125 & \multirow[b]{2}{*}{$\leq 0.062$} & \multirow[b]{2}{*}{0.125} \\
\hline & MBC & $39(75)$ & $9(17)$ & $1(2)$ & & $1(2)$ & $1(2)$ & $1(2)$ & & & & \\
\hline \multirow{2}{*}{ M. abscessus (64) } & MIC & & $2(3)$ & $20(31)$ & $42(66)$ & & & & 0.5 & 0.5 & \multirow[b]{2}{*}{0.5} & \multirow[b]{2}{*}{0.5} \\
\hline & MBC & & & $2(3)$ & $58(91)$ & $4(6)$ & & & & & & \\
\hline \multirow{2}{*}{ M. massiliense (67) } & MIC & $2(3)$ & $4(6)$ & $51(76)$ & $10(15)$ & & & & 0.25 & 0.5 & \multirow[b]{2}{*}{0.5} & \multirow[b]{2}{*}{0.5} \\
\hline & MBC & $1(2)$ & & $25(37)$ & $41(61)$ & & & & & & & \\
\hline
\end{tabular}

* Data are presented as number (\%). MIC: minimum inhibitory concentration; MBC: minimum bactericidal concentration; NTM: nontuberculous mycobacteria.

The MIC of clofazimine for MAC or M. kansasii tended to be lower than that of M. abscessus or M. massiliense. The $\mathrm{MIC}_{50}$ and $\mathrm{MIC}_{90}$ of clofazimine against $M$. kansasii were $\leq 0.062 \mu \mathrm{g} / \mathrm{mL}$ and $0.125 \mu \mathrm{g} / \mathrm{mL}$, respectively, showing the lowest values among all NTM species. The $\mathrm{MIC}_{50}$ and $\mathrm{MIC}_{90}$ of clofazimine for $M$. abscessus were the highest at $0.5 \mu \mathrm{g} / \mathrm{mL}$. The MBC values of clofazimine in each NTM species showed similar trends to the MIC values.

\subsection{In Vitro Activity of Clofazimine in NTM with Drug Resistance}

Clofazimine MICs and MBCs were also determined against 57 clarithromycin- and 35 amikacin-resistant NTM (Table 2), which were identified through sequence analysis for mutations associated with acquired resistance to clarithromycin and amikacin, respectively. Overall, the clofazimine MICs were low in all NTM species regardless of resistance to clarithromycin or amikacin. For clarithromycin-resistant isolates, most of the isolates, $82 \%(47 / 57)$, had clofazimine MICs $\leq 0.25 \mu \mathrm{g} / \mathrm{mL}$, including all $(10 / 10)$ of M. avium, $88 \%(15 / 17)$ of M. intracellulare, $62 \%(8 / 13)$ of M. abscessus, and $82 \%(14 / 17)$ of M. massiliense. The $\mathrm{MIC}_{50}$ and $\mathrm{MIC}_{90}$ values of clofazimine were $0.25 \mu \mathrm{g} / \mathrm{mL}$ and $0.5 \mu \mathrm{g} / \mathrm{mL}$ in $M$. abscessus and M. massiliense, respectively, higher than those in M. avium or M. intracellulare. 
Table 2. $\mathrm{MIC}_{50}, \mathrm{MIC}_{90}, \mathrm{MBC}_{50}$, and $\mathrm{MBC}_{90}$ values of clofazimine for 57 clarithromycin-resistant and 35 amikacin-resistant NTM isolates *.

\begin{tabular}{|c|c|c|c|c|c|c|c|c|c|c|}
\hline \multirow{2}{*}{ NTM Species (No.) } & \multirow{2}{*}{ Activity } & \multicolumn{5}{|c|}{ No. $(\%)$ of Isolates According to MIC $(\mu \mathrm{g} / \mathrm{mL})^{*}$} & \multirow{2}{*}{$\begin{array}{c}\mathrm{MIC}_{50} \\
(\mu \mathrm{g} / \mathrm{mL})\end{array}$} & \multirow{2}{*}{$\begin{array}{c}\mathrm{MIC}_{90} \\
(\mu \mathrm{g} / \mathrm{mL})\end{array}$} & \multirow{2}{*}{$\begin{array}{l}\mathrm{MBC}_{50} \\
(\mu \mathrm{g} / \mathrm{mL})\end{array}$} & \multirow{2}{*}{$\begin{array}{l}\mathrm{MBC}_{90} \\
(\mu \mathrm{g} / \mathrm{mL})\end{array}$} \\
\hline & & $\leq 0.062$ & 0.125 & 0.25 & 0.5 & 1 & & & & \\
\hline \multicolumn{11}{|l|}{ Clarithromycin-resistant } \\
\hline \multirow{2}{*}{ M. avium (10) } & MIC & $4(40)$ & $6(60)$ & & & & 0.125 & 0.125 & & \\
\hline & MBC & $3(30)$ & $5(50)$ & $2(20)$ & & & & & 0.125 & 0.25 \\
\hline \multirow{2}{*}{ M. intracellulare (17) } & MIC & $3(17)$ & $8(47)$ & $4(24)$ & $2(12)$ & & 0.125 & 0.5 & & \\
\hline & MBC & $3(17)$ & $5(30)$ & $5(30)$ & $3(17)$ & $1(6)$ & & & 0.25 & 0.5 \\
\hline \multirow{2}{*}{ M. abscessus (13) } & MIC & & $2(15)$ & $6(46)$ & $4(31)$ & $1(8)$ & 0.25 & 0.5 & & \\
\hline & $\mathrm{MBC}$ & & $1(8)$ & $4(31)$ & $7(53)$ & $1(8)$ & & & 0.5 & 0.5 \\
\hline \multirow{2}{*}{ M. massiliense (17) } & MIC & & $6(35)$ & $8(47)$ & $3(18)$ & & 0.25 & 0.5 & & \\
\hline & MBC & & & $7(42)$ & $10(58)$ & & & & 0.5 & 0.5 \\
\hline \multicolumn{11}{|l|}{ Amikacin-resistant } \\
\hline \multirow{2}{*}{ M. avium (11) } & MIC & $11(100)$ & & & & & $\leq 0.062$ & $\leq 0.062$ & & \\
\hline & MBC & $11(100)$ & & & & & & & $\leq 0.062$ & $\leq 0.062$ \\
\hline \multirow{2}{*}{ M. intracellulare (15) } & MIC & $9(60)$ & $3(20)$ & $2(13)$ & $1(7)$ & & $\leq 0.062$ & 0.25 & & \\
\hline & MBC & $7(46)$ & $4(27)$ & $3(20)$ & $1(7)$ & & & & 0.125 & 0.25 \\
\hline \multirow{2}{*}{ M. abscessus (6) } & MIC & & & $5(83)$ & $1(17)$ & & 0.25 & 0.5 & & \\
\hline & $\mathrm{MBC}$ & & & & $6(100)$ & & & & 0.5 & 0.5 \\
\hline \multirow{2}{*}{ M. massiliense (3) } & MIC & & & $2(67)$ & $1(33)$ & & 0.5 & 0.5 & & \\
\hline & MBC & & & $1(33)$ & $2(67)$ & & & & 0.5 & 0.5 \\
\hline
\end{tabular}

* Data are presented as number (\%). MIC: minimum inhibitory concentration; MBC: minimum bactericidal concentration; NTM: nontuberculous mycobacteria.

For amikacin-resistant isolates, all $M$. avium isolates showed the lowest clofazimine MICs, $\leq 0.062 \mu \mathrm{g} / \mathrm{mL}$. Clofazimine MICs of $\leq 0.25 \mu \mathrm{g} / \mathrm{mL}$ were found for $93 \%(14 / 15)$ of M. intracellulare, $83 \%(5 / 6)$ of $M$. abscessus, and $67 \%(2 / 3)$ of $M$. massiliense isolates. MIC $_{50}$ and MIC $_{90}$ values in M. abscessus or M. massiliense were higher than those in M. avium or M. intracellulare. Overall, clofazimine MBC and MIC values showed similar trends.

\subsection{Characteristics of Patients Treated with Clofazimine-Containing Regimens}

For the 58 patients treated with clofazimine-containing regimens for $\geq 6$ months, clinical characteristics were evaluated at the regimen start time (Table 3). Of them, 40 patients received clofazimine for $\geq 12$ months, and 18 patients received clofazimine for $6-12$ months. The median age was 61 years, and $69 \%$ of patients were female. The most common underlying condition was a previous history of pulmonary tuberculosis $(50 \%)$, followed by chronic pulmonary aspergillosis (10\%). The most common etiologic organism was M. abscessus (60\%), followed by M. massiliense (26\%). The nodular bronchiectatic form of NTM-PD was present in $37(64 \%)$ patients, with $(15 / 37)$ or without $(22 / 37)$ cavity, and the remaining $21(36 \%)$ had the fibrocavitary form. Isolates resistant to clarithromycin or amikacin were confirmed in 20 patients, 14 of whom had M. abscessus-PD or M. massiliense-PD; the remaining six had $M$. avium-PD or $M$. intracellulare-PD.

Table 3. Characteristics of patients treated with clofazimine-containing regimens $(n=58)$.

\begin{tabular}{cc}
\hline Characteristics & Values \\
\hline Age, years & $61(55-69)$ \\
Sex, female & $40(69)$ \\
Body mass index, $\mathrm{kg} / \mathrm{m}^{2}$ & $20.9(18.1-22.2)$ \\
Never-smoker & $43(74)$ \\
Comorbidity & $29(50)$ \\
Previous history of pulmonary tuberculosis & $6(10)$ \\
Chronic pulmonary aspergillosis & $5(9)$ \\
Obstructive pulmonary disease & $5(9)$ \\
Diabetes mellitus &
\end{tabular}


Table 3. Cont.

\begin{tabular}{cc}
\hline Characteristics & Values \\
\hline Chronic heart disease & $4(7)$ \\
Chronic liver disease & $2(3)$ \\
Previous lung cancer & $1(2)$ \\
Other malignancy * & $2(3)$ \\
Etiologic organism & \\
M. abscessus & $35(60)$ \\
M. massiliense & $15(26)$ \\
M. avium & $3(5)$ \\
M. intracellulare & $5(9)$ \\
Radiologic findings & $37(64)$ \\
Nodular bronchiectatic form & $15 / 37$ \\
With cavity & $22 / 37$ \\
Without cavity & $21(36)$ \\
Fibrocavitary form & \\
Laboratory findings & $41(71)$ \\
Positive sputum acid-fast bacilli smear & $55.0(24.3-68.3)$ \\
Erythrocyte sedimentation rate, mm/h & $20(34)$ \\
Clarithromycin or amikacin resistance $\mathbb{I}$ & $14 / 20$ \\
M. abscessus or M. massiliense & $6 / 20$ \\
M. avium or M. intracellulare &
\end{tabular}

Data are presented as number (\%) or median (interquartile range). ${ }^{*}$ Rectal cancer $(n=1)$, sarcoma $(n=1)$ II Isolates resistant to macrolide or aminoglycoside or both.

\subsection{Treatment Outcome at 12 Months after Clofazimine-Containing Regimens}

Table 4 shows the culture conversion data of 38 NTM-PD patients without drug-resistant isolates. Sputum culture conversion was achieved in $47 \%(18 / 38)$ of patients, including 8/27 of M. abscessus, 9/9 of M. massiliense, $0 / 1$ of $M$. avium, and 1/1 of M. intracellulare (Supplementary Figure S1). Regardless of NTM species, as the MIC decreased, the conversion rate tended to be higher (Supplementary Figure S2A, $p=0.004$ ). Also, the conversion rate was significantly higher in patients with MICs $\leq 0.25 \mu \mathrm{g} / \mathrm{mL}$ than with MICs $=0.5 \mu \mathrm{g} / \mathrm{mL}$ (Supplementary Figure S2B, $p=0.004$ ). After adjusting for various clinical, laboratory, and demographic factors, clofazimine MICs $\leq 0.25 \mu \mathrm{g} / \mathrm{mL}$ remained a significant factor associated with culture conversion (Table 5).

Table 4. Culture conversion rate after 12 months of treatment in patients without drug-resistant isolates according to MIC value and NTM species $(n=38)$.

\begin{tabular}{|c|c|c|c|c|c|c|c|c|c|c|c|}
\hline \multirow[b]{2}{*}{ MIC } & \multicolumn{5}{|c|}{ No Conversion } & \multicolumn{5}{|c|}{ Conversion } & \multirow[b]{2}{*}{$p$-Value } \\
\hline & $\begin{array}{c}\text { M. ab- } \\
\text { scessus }\end{array}$ & $\begin{array}{l}\text { M. mas- } \\
\text { siliense }\end{array}$ & $\begin{array}{c}\text { M. } \\
\text { avium }\end{array}$ & $\begin{array}{l}\text { M. in- } \\
\text { tracel- } \\
\text { lulare }\end{array}$ & Total * & $\begin{array}{l}\text { M. } a b- \\
\text { scessus }\end{array}$ & $\begin{array}{l}\text { M. mas- } \\
\text { siliense }\end{array}$ & $\begin{array}{c}\text { M. } \\
\text { avium }\end{array}$ & $\begin{array}{l}\text { M. in- } \\
\text { tracel- } \\
\text { lulare }\end{array}$ & Total II & \\
\hline$\leq 0.062$ & - & - & - & - & - & - & 1 & - & - & 1 & \multirow{4}{*}{0.004} \\
\hline 0.125 & - & - & 1 & - & 1 & - & - & - & - & - & \\
\hline 0.25 & 4 & - & - & - & 4 & 3 & 8 & - & 1 & 12 & \\
\hline 0.5 & 15 & - & - & - & 15 & 5 & - & - & - & 5 & \\
\hline MIC & $\begin{array}{l}\text { M. ab- } \\
\text { scessus }\end{array}$ & $\begin{array}{l}\text { M. mas- } \\
\text { siliense }\end{array}$ & $\begin{array}{c}\text { M. } \\
\text { avium }\end{array}$ & $\begin{array}{l}\text { M. intra- } \\
\text { cellulare }\end{array}$ & Total * & $\begin{array}{l}\text { M. ab- } \\
\text { scessus }\end{array}$ & $\begin{array}{l}\text { M. mas- } \\
\text { siliense }\end{array}$ & $\begin{array}{c}\text { M. } \\
\text { avium }\end{array}$ & $\begin{array}{l}\text { M. intra- } \\
\text { cellulare }\end{array}$ & Total II & $p$-value \\
\hline$\leq 0.25$ & 4 & - & 1 & - & 5 & 3 & 9 & - & 1 & 13 & \multirow{2}{*}{0.004} \\
\hline$\geq 0.5$ & 15 & - & - & - & 15 & 5 & - & - & - & 5 & \\
\hline
\end{tabular}

Data are presented as number. $p$-value is the value comparing Total * and Total ${ }^{\mathbb{I}}$ regardless of NTM species. MIC: minimum inhibitory concentration; NTM: nontuberculous mycobacteria. 
Table 5. Factors associated with culture conversion at 12 months in patients without drug-resistant isolates $(n=38)$.

\begin{tabular}{|c|c|c|c|c|}
\hline \multirow[b]{2}{*}{ Variable } & \multicolumn{2}{|c|}{ Univariable } & \multicolumn{2}{|c|}{ Multivariable } \\
\hline & $\begin{array}{l}\text { Unadjusted HR } \\
(95 \% \mathrm{CI})\end{array}$ & $p$ Value & $\begin{array}{l}\text { Adjusted HR } \\
\quad(95 \% \mathrm{CI})\end{array}$ & $p$ Value \\
\hline Age $<65$ years & $1.500(0.534-4.214)$ & 0.442 & $3.099(0.680-14.129)$ & 0.144 \\
\hline Female & $0.626(0.206-1.909)$ & 0.411 & - & - \\
\hline Body mass index $\geq 18.5 \mathrm{~kg} / \mathrm{m}^{2}$ & $1.496(0.561-3.990)$ & 0.421 & $1.452(0.374-5.631)$ & 0.590 \\
\hline Never smoker & $1.347(0.442-4.102)$ & 0.600 & - & - \\
\hline $\begin{array}{c}\text { No previous pulmonary tuberculosis } \\
\text { Etiology }\end{array}$ & $0.825(0.326-2.092)$ & 0.686 & $0.953(0.232-3.906)$ & 0.947 \\
\hline M. abscessus & Reference & & Reference & \\
\hline M. massiliense & $9.179(3.116-27.040)$ & $<0.001$ & $51.978(1.463-1846.164)$ & 0.030 \\
\hline M. avium/M. intracellulare & $1.373(0.172-10.987)$ & 0.765 & $0.214(0.004-11.608)$ & 0.449 \\
\hline Negative sputum acid-fast bacilli smear & $1.738(0.681-4.431)$ & 0.247 & $1.521(0.416-5.564)$ & 0.526 \\
\hline No cavity & $0.877(0.329-2.339)$ & 0.793 & $130.124(4.423-3827.900)$ & 0.005 \\
\hline \multicolumn{5}{|l|}{ MIC of clofazimine, $\mu \mathrm{g} / \mathrm{mL}$} \\
\hline$\geq 0.5$ & reference & & reference & \\
\hline$\leq 0.25$ & $4.382(1.548-12.408)$ & 0.005 & $22.458(1.595-316.251)$ & 0.021 \\
\hline \multicolumn{5}{|l|}{ Macrolide resistance } \\
\hline Inducible resistance & Reference & & Reference & \\
\hline Susceptible & $3.452(1.325-8.995)$ & 0.011 & $0.274(0.018-4.105)$ & 0.349 \\
\hline Intravenous amikacin use, days & $0.974(0.944-1.005)$ & 0.095 & $0.956(0.900-1.016)$ & 0.149 \\
\hline Elevated erythrocyte sedimentation rate * & $0.292(0.039-2.195)$ & 0.232 & $0.754(0.067-8.424)$ & 0.818 \\
\hline
\end{tabular}

HR: hazard ratio; CI: confidential interval; MIC: minimum inhibitory concentration. * Erythrocyte sedimentation rate $>15 \mathrm{~mm} / \mathrm{h}(\mathrm{men})$ or $>20 \mathrm{~mm} / \mathrm{h}$ (women).

For the 20 NTM-PD patients with clarithromycin- or amikacin-resistant isolates, culture conversion was achieved in $20 \%(4 / 20)$, including $3 / 14$ of $M$. abscessus and $1 / 6$ of M. intracellulare (Supplementary Figure S3). However, the conversion rate according to MIC value was not statistically significant.

Overall, as MIC decreased, the conversion rate among all 58 NTM-PD patients trended higher, but the trend was not statistically significant (Supplementary Figure S4A,B). Furthermore, after adjusting for various factors, MICs $\leq 0.25 \mu \mathrm{g} / \mathrm{mL}$ of clofazimine were not a significant factor associated with culture conversion (Supplementary Table S2).

\section{Discussion}

This study revealed that clofazimine was effective in vitro against major pathogenic NTM species, regardless of resistance to key antibiotics such as clarithromycin or amikacin. In addition, our data showed that lower MICs of clofazimine were associated with higher culture conversion rates at 12 months after treatment in NTM-PD patients, especially for NTM without resistance to key antibiotics. Thus, our data imply that clofazimine could be a viable therapeutic option for patients with NTM-PD.

Our data showed that $80-90 \%$ of NTM isolates had clofazimine MICs $\leq 0.25 \mu \mathrm{g} / \mathrm{mL}$ regardless of drug resistance pattern, except for M. abscessus, for which $34 \%$ of drugsusceptible isolates and $62 \%$ of isolates with clarithromycin and/or amikacin resistance had clofazimine MICs $\leq 0.25 \mu \mathrm{g} / \mathrm{mL}$. Notably, MIC or MBC values in M. kansasii were the lowest among all the NTM species in our study. Similar to our present findings, previous studies analyzing in vitro activity of clofazimine have shown a generally low distribution of MIC values across NTM species with various MIC levels [18,22-25]. In a Taiwanese study evaluating RGM, approximately $99 \%$ of $117 \mathrm{M}$. abscessus isolates had clofazimine MICs $\leq 1 \mu \mathrm{g} / \mathrm{mL}$ [22]. Jakko et al. demonstrated $\mathrm{MIC}_{50}$ and $\mathrm{MIC}_{90}$ of $\leq 0.5 \mu \mathrm{g} / \mathrm{mL}$ and $1.0 \mu \mathrm{g} / \mathrm{mL}$, respectively, in a study that evaluated synergy between clofazimine and amikacin using 342 M. abscessus isolates [18]. In a MAC study from China, clofazimine demonstrated strong activity with tentative epidemiological cutoffs at $1 \mu \mathrm{g} / \mathrm{mL}$ and $2 \mu \mathrm{g} / \mathrm{mL}$ for $\mathrm{MIC}_{50}$ and $\mathrm{MIC}_{90}$, respectively [25]. Nevertheless, a more remarkable finding of our study is that, despite clarithromycin or amikacin resistance, most NTM isolates 
had low clofazimine MIC or MBC values across NTM species. For example, all (10/10) clarithromycin-resistant $M$. avium and 83\% (5/6) of amikacin-resistant $M$. abscessus had clofazimine MICs $\leq 0.25 \mu \mathrm{g} / \mathrm{mL}$. We also found low MIC values for $M$. kansasii, which has not been reported in previous studies. In this context, given that there are limited data on the in vitro activity of clofazimine using large sample sizes and various types of clinical NTM strains, our results provide important insights.

A major challenge in managing NTM-PD, especially refractory PD, is the lack of sufficient and appropriate active drugs that can be used throughout long-term maintenance therapy. For MAC-PD, although guidelines recommend macrolide-based multidrug therapy including intravenous- or liposomal-inhaled amikacin based on the results of DST $[4,5,26]$, no effective oral agent has been shown other than rifampin and ethambutol. Especially for M. abscessus-PD, the pathogen is highly drug-resistant, and long-term use of injectable antibiotics is not feasible in real-world practice. The efficacy of liposomal-inhaled amikacin in treatment of MAC-PD has not been proven in M. abscessus-PD. Moreover, only clarithromycin and amikacin have demonstrated a correlation between clinical outcome and in vitro activity. Thus, there is a need for research on drugs that can be selected when guideline-recommended drugs become unavailable or ineffective due to adverse effects or resistance. Given this context, our results suggest that clofazimine could be considered, depending on MIC level, in NTM-PD treatment.

Another important finding of our study was that lower clofazimine MIC values were associated with culture conversion in NTM-PD patients. This phenomenon was more clearly observed in 38 NTM-PD patients without resistance to key antibiotics such as clarithromycin or amikacin. As the MIC decreased, the conversion rate increased and was significantly higher in patients with MICs $\leq 0.25 \mu \mathrm{g} / \mathrm{mL}$ than in those with MIC $=0.5 \mu \mathrm{g} / \mathrm{mL}$ of clofazimine (Supplementary Figure S2). Moreover, an MIC $\leq 0.25 \mu \mathrm{g} / \mathrm{mL}$ remained a significant factor in multivariable analysis (Table 5). However, when adding patients affected by drug-resistant NTM to the above analysis, the association between lower MIC and culture conversion was not significant (Supplementary Figure S4 and Supplementary Table S2). These findings imply that clarithromycin or amikacin susceptibility is critical in treatment of NTM-PD, in all combinations of clofazimine and other agents. Indeed, prior studies have indicated poor treatment outcomes after the occurrence of acquired macrolide resistance in MAC- or M. abscessus-PD patients [27-30]. Previous studies have reported unsatisfactory clinical outcomes of salvage clofazimine treatment in refractory MAC- or M. abscessus-PD, with a less than 30\% conversion rate [12,13]. Additionally, given the synergism between clofazimine and amikacin (or clarithromycin) $[18,24,31,32]$, it is estimated that this strategy will not be effective in cases of resistance to clarithromycin or amikacin. Thus, more data on the clinical applicability of clofazimine are needed.

Interestingly, in our study, among the 27 patients with M. abscessus without acquired drug resistance to clarithromycin or amikacin, culture conversion rates tended to be higher in patient groups with low MICs of clofazimine $(43 \%(3 / 7)$ of the MIC $=0.25 \mu \mathrm{g} / \mathrm{mL}$ group vs. $25 \%(5 / 20)$ of the MIC $=0.5 \mu \mathrm{g} / \mathrm{mL}$ group, Supplementary Figure S1). This finding suggests that, when M. abscessus shows a low clofazimine MIC without drug resistance against a macrolide or aminoglycoside, clofazimine is worth considering as a therapeutic agent. As noted previously, clofazimine has several advantages for treatment of NTM-PD. However, to date, limited data exist on the pharmacokinetics of clofazimine, and there is no consensus on the optimal dosage or administration interval. Thus, further studies are warranted on its use for NTM-PD.

There are several limitations to our study. First, our MIC data might not be generalizable to other geographic areas. Second, several factors might have influenced the clinical outcome. As the outcome was mainly evaluated after 12 months, the final treatment outcome might have differed if the treatment period was extended. In addition, some patients received clofazimine for less than 12 months, and the number of patients included in the clinical outcome analysis and multivariate analysis was small. Lastly, we did not evaluate the mechanism associated with high MIC of clofazimine in NTM species. 
Zhang et al. showed that $97 \%$ of clofazimine-resistant $M$. tuberculosis strains had mutations in Rv0678 [33]; however, limited data are available on the association between gene mutations and resistance to clofazimine in NTM species. In a recent Chinese study that evaluated in vitro susceptibilities to clofazimine of clinical NTM isolates, there was no specific mutation within the Rv0678 among $22 \mathrm{M}$. avium isolates, and only 2/35 M. intracellulare isolates with MICs $\geq 8 \mu \mathrm{g} / \mathrm{mL}$ had Rv0678 mutation [25]. Further studies on these topics are needed.

In conclusion, we found that clofazimine had effective in vitro activity for major pathogenic NTM species regardless of resistance to key antibiotics such as clarithromycin or amikacin. Given that lower MIC values of clofazimine are associated with higher culture conversion rates at 12 months after treatment in NTM-PD patients without drug resistance, clofazimine could be a potential therapeutic option for patients with NTM-PD. However, further research on the effect of clofazimine in patients with isolates resistant to key antibiotics such as clarithromycin and amikacin is necessary.

Supplementary Materials: The following are available online at https:/ /www.mdpi.com/article/10 $.3390 / \mathrm{jcm} 10194581 / \mathrm{s} 1$, Figure S1: Culture conversion rate after 12 months of treatment in NTM-PD patients without drug-resistant isolates according to MIC value of clofazimine and NTM species $(n=38)$, Figure S2: (A) Culture conversion rate after 12 months of treatment in NTM-PD patients without drug-resistant isolates according to MIC value of clofazimine, regardless of NTM species $(n=38)$, (B) Culture conversion rate after 12 months of treatment in NTM-PD patients without drugresistant isolates according to clofazimine MIC of $0.25 \mu \mathrm{g} / \mathrm{mL}$, regardless of NTM species ( $n=38)$, Figure S3: Culture conversion rate after 12 months of treatment in NTM-PD patients with drugresistant isolates $(n=20)$, Figure S4: (A) Culture conversion rate after 12 months of treatment in all study patients according to MIC value of clofazimine, regardless of NTM species $(n=58)$, (B) Culture conversion rate after 12 months of treatment in all study patients according to clofazimine MIC of $0.25 \mu \mathrm{g} / \mathrm{mL}$, regardless of NTM species $(n=58)$, Table S1: Companion drugs used with clofazimine, Table S2: Factors associated with culture conversion in patients treated with clofazimine-containing regimens $(n=58)$.

Author Contributions: Study conception and design, D.H.K., B.W.J. and W.-J.K.; data acquisition and analysis, D.H.K., B.-G.K., S.-Y.K., H.J.H., N.Y.L., H.K., and O.J.K.; data interpretation and manuscript writing: D.H.K., B.-G.K. and B.W.J. All authors have read and agreed to the published version of the manuscript.

Funding: This research was supported by a grant of the Korea Health Technology R\&D Project through the Korea Health Industry Development Institute (KHIDI), funded by the Ministry of Health and Welfare, Korea (HI20C0017).

Institutional Review Board Statement: All NTM isolates and the clinical data included in this study were obtained from an Institutional Review Board-approved observational cohort at Samsung Medical Center (ClinicalTrials.gov identifier: NCT00970801).

Informed Consent Statement: Informed consent was obtained from all subjects involved in the study.

Acknowledgments: We would like to express our heartfelt gratitude and respect to Won-Jung Koh for his invaluable guidance and unfailing support throughout this research. Won-Jung Koh passed away in August 2019. We dedicate this work to his memory.

Conflicts of Interest: The authors have no conflict of interest to declare.
Abbreviations
PD
pulmonary disease
NTM nontuberculous mycobacteria
MAC mycobacterium avium complex
DST drug susceptibility testing
CLSI Clinical and Laboratory Standards Institute
RGM rapidly growing mycobacteria 
SGM slowly growing mycobacteria

MIC minimum inhibitory concentration

MBC minimum bactericidal concentration

\section{References}

1. Prevots, D.R.; Marras, T.K. Epidemiology of human pulmonary infection with nontuberculous mycobacteria: A review. Clin. Chest Med. 2015, 36, 13-34. [CrossRef] [PubMed]

2. Lee, H.; Myung, W.; Koh, W.J.; Moon, S.M.; Jhun, B.W. Epidemiology of nontuberculous mycobacterial infection, South Korea, 2007-2016. Emerg. Infect. Dis. 2019, 25, 569-572. [CrossRef]

3. Hoefsloot, W.; van Ingen, J.; Andrejak, C.; Angeby, K.; Bauriaud, R.; Bemer, P.; Beylis, N.; Boeree, M.J.; Cacho, J.; Chihota, V.; et al. The geographic diversity of nontuberculous mycobacteria isolated from pulmonary samples: An NTM-NET collaborative study. Eur. Respir. J. 2013, 42, 1604-1613. [CrossRef]

4. Daley, C.L.; Iaccarino, J.M.; Lange, C.; Cambau, E.; Wallace, R.J.; Andrejak, C.; Böttger, E.C.; Brozek, J.; Griffith, D.E.; Guglielmetti, L.; et al. Treatment of nontuberculous mycobacterial pulmonary disease: An Official ATS/ERS/ESCMID/IDSA clinical practice guideline. Clin. Infect. Dis. 2020, 71, 905-913. [CrossRef] [PubMed]

5. Haworth, C.S.; Banks, J.; Capstick, T.; Fisher, A.J.; Gorsuch, T.; Laurenson, I.F.; Leitch, A.; Loebinger, M.R.; Milburn, H.J.; Nightingale, M.; et al. British Thoracic Society guidelines for the management of non-tuberculous mycobacterial pulmonary disease (NTM-PD). Thorax 2017, 72, ii1-ii64. [CrossRef] [PubMed]

6. Kwak, N.; Park, J.; Kim, E.; Lee, C.H.; Han, S.K.; Yim, J.J. Treatment outcomes of Mycobacterium avium complex lung disease: A systematic review and meta-analysis. Clin. Infect. Dis. 2017, 65, 1077-1084. [CrossRef] [PubMed]

7. Kwak, N.; Dalcolmo, M.P.; Daley, C.L.; Eather, G.; Gayoso, R.; Hasegawa, N.; Jhun, B.W.; Koh, W.J.; Namkoong, H.; Park, J.; et al. Mycobacterium abscessus pulmonary disease: Individual patient data meta-analysis. Eur. Respir. J. 2019, 54, 1801991. [CrossRef] [PubMed]

8. Kim, S.Y.; Kim, C.K.; Bae, I.K.; Jeong, S.H.; Yim, J.J.; Jung, J.Y.; Park, M.S.; Kim, Y.S.; Kim, S.K.; Chang, J.; et al. The drug susceptibility profile and inducible resistance to macrolides of Mycobacterium abscessus and Mycobacterium massiliense in Korea. Diagn. Microbiol. Infect. Dis. 2015, 81, 107-111. [CrossRef]

9. Martiniano, S.L.; Wagner, B.D.; Levin, A.; Nick, J.A.; Sagel, S.D.; Daley, C.L. Safety and effectiveness of clofazimine for primary and refractory nontuberculous mycobacterial Infection. Chest 2017, 152, 800-809. [CrossRef]

10. Jarand, J.; Davis, J.P.; Cowie, R.L.; Field, S.K.; Fisher, D.A. Long-term follow-up of Mycobacterium avium complex lung disease in patients treated with regimens including clofazimine and/or rifampin. Chest 2016, 149, 1285-1293. [CrossRef]

11. Field, S.K.; Cowie, R.L. Treatment of Mycobacterium avium-intracellulare complex lung disease with a macrolide, ethambutol, and clofazimine. Chest 2003, 124, 1482-1486. [CrossRef]

12. Kim, B.G.; Kim, H.; Kwon, O.J.; Huh, H.J.; Lee, N.Y.; Baek, S.Y.; Sohn, I.; Jhun, B.W. Outcomes of inhaled amikacin and clofazimine-containing regimens for treatment of refractory Mycobacterium avium complex pulmonary disease. J. Clin. Med. 2020, 9, 2968. [CrossRef]

13. Yang, B.; Jhun, B.W.; Moon, S.M.; Lee, H.; Park, H.Y.; Jeon, K.; Kim, D.H.; Kim, S.Y.; Shin, S.J.; Daley, C.L.; et al. Clofaziminecontaining regimen for the treatment of Mycobacterium abscessus lung disease. Antimicrob. Agents Chemother. 2017, 61, e02052-16. [CrossRef] [PubMed]

14. Cholo, M.C.; Steel, H.C.; Fourie, P.B.; Germishuizen, W.A.; Anderson, R. Clofazimine: Current status and future prospects. J. Antimicrob. Chemother. 2012, 67, 290-298. [CrossRef]

15. Aung, K.J.; van Deun, A.; Declercq, E.; Sarker, M.R.; Das, P.K.; Hossain, M.A.; Rieder, H.L. Successful 9-month Bangladesh regimen' for multidrug-resistant tuberculosis among over 500 consecutive patients. Int. J. Tuberc. Lung Dis. 2014, 18, 1180-1187. [CrossRef]

16. Wang, Q.; Pang, Y.; Jing, W.; Liu, Y.; Wang, N.; Yin, H.; Zhang, Q.; Ye, Z.; Zhu, M.; Li, F.; et al. Clofazimine for treatment of extensively drug-resistant pulmonary tuberculosis in China. Antimicrob. Agents Chemother. 2018, 62, e02149. [CrossRef] [PubMed]

17. Reddy, V.M.; O'Sullivan, J.F.; Gangadharam, P.R. Antimycobacterial activities of riminophenazines. J. Antimicrob. Chemother. 1999, 43, 615-623. [CrossRef] [PubMed]

18. Van Ingen, J.; Totten, S.E.; Helstrom, N.K.; Heifets, L.B.; Boeree, M.J.; Daley, C.L. In vitro synergy between clofazimine and amikacin in treatment of nontuberculous mycobacterial disease. Antimicrob. Agents Chemother. 2012, 56, 6324-6327. [CrossRef] [PubMed]

19. Clinical Laboratory Standards Institute (CLSI). Susceptibility Testing of Mycobacteria, Nocardia spp., and other Aerobic Actinomycetes; Approved Standard, 3rd ed.; CLSI document No. M24; Clinical Laboratory Standards Institute: Wayne, PA, USA, 2018.

20. Clinical Laboratory Standards Institute (CLSI). Performance Standards for Susceptibility Testing of Mycobacteria, Nocardia spp., and other Aerobic Actinomycetes; Approved Standard, 1st ed.; CLSI document No. M62; Clinical Laboratory Standards Institute: Wayne, PA, USA, 2018.

21. Nessar, R.; Cambau, E.; Reyrat, J.M.; Murray, A.; Gicquel, B. Mycobacterium abscessus: A new antibiotic nightmare. J. Antimicrob. Chemother. 2012, 67, 810-818. [CrossRef] 
22. Huang, C.C.; Wu, M.F.; Chen, H.C.; Huang, W.C. In vitro activity of aminoglycosides, clofazimine, d-cycloserine and dapsone against 83 Mycobacterium avium complex clinical isolates. J. Microbiol. Immunol. Infect. 2018, 51, 636-643. [CrossRef]

23. Singh, S.; Bouzinbi, N.; Chaturvedi, V.; Godreuil, S.; Kremer, L. In vitro evaluation of a new drug combination against clinical isolates belonging to the Mycobacterium abscessus complex. Clin. Microbiol. Infect. 2014, 20, O1124-O1127. [CrossRef] [PubMed]

24. Schwartz, M.; Fisher, S.; Story-Roller, E.; Lamichhane, G.; Parrish, N. Activities of dual combinations of antibiotics against multidrug-resistant nontuberculous mycobacteria recovered from patients with cystic fibrosis. Microb. Drug Resist. 2018, 24, 1191-1197. [CrossRef] [PubMed]

25. Luo, J.; Yu, X.; Jiang, G.; Fu, Y.; Huo, F.; Ma, Y.; Wang, F.; Shang, Y.; Liang, Q.; Xue, Y.; et al. In vitro activity of clofazimine against nontuberculous mycobacteria isolated in Beijing, China. Antimicrob. Agents Chemother. 2018, 62, e00072-18. [CrossRef] [PubMed]

26. Griffith, D.E.; Aksamit, T.; Brown-Elliott, B.A.; Catanzaro, A.; Daley, C.; Gordin, F.; Holland, S.M.; Horsburgh, R.; Huitt, G.; Iademarco, M.F.; et al. An official ATS/IDSA statement: Diagnosis, treatment, and prevention of nontuberculous mycobacterial diseases. Am. J. Respir. Crit. Care Med. 2007, 175, 367-416. [CrossRef]

27. Griffith, D.E.; Brown-Elliott, B.A.; Langsjoen, B.; Zhang, Y.; Pan, X.; Girard, W.; Nelson, K.; Caccitolo, J.; Alvarez, J.; Shepherd, S.; et al. Clinical and molecular analysis of macrolide resistance in Mycobacterium avium complex lung disease. Am. J. Respir. Crit. Care Med. 2006, 174, 928-934. [CrossRef]

28. Moon, S.M.; Park, H.Y.; Kim, S.Y.; Jhun, B.W.; Lee, H.; Jeon, K.; Kim, D.H.; Huh, H.J.; Ki, C.S.; Lee, N.Y.; et al. Clinical characteristics, treatment outcomes, and resistance mutations associated with macrolide-resistant Mycobacterium avium complex lung disease. Antimicrob. Agents Chemother. 2016, 60, 6758-6765. [CrossRef]

29. Morimoto, K.; Namkoong, H.; Hasegawa, N.; Nakagawa, T.; Morino, E.; Shiraishi, Y.; Ogawa, K.; Izumi, K.; Takasaki, J.; Yoshiyama, T.; et al. Macrolide-resistant Mycobacterium avium complex lung disease: Analysis of 102 consecutive cases. Ann. Am. Thorac. Soc. 2016, 13, 1904-1911. [CrossRef]

30. Choi, H.; Kim, S.Y.; Kim, D.H.; Huh, H.J.; Ki, C.S.; Lee, N.Y.; Lee, S.H.; Shin, S.; Shin, S.J.; Daley, C.L.; et al. Clinical characteristics and treatment outcomes of patients with acquired macrolide-resistant Mycobacterium abscessus lung disease. Antimicrob. Agents Chemother. 2017, 61, e01146. [CrossRef]

31. Shen, G.H.; Wu, B.D.; Hu, S.T.; Lin, C.F.; Wu, K.M.; Chen, J.H. High efficacy of clofazimine and its synergistic effect with amikacin against rapidly growing mycobacteria. Int. J. Antimicrob. Agents 2010, 35, 400-404. [CrossRef]

32. Lanoix, J.P.; Joseph, C.; Peltier, F.; Castelain, S.; Andréjak, C. Synergistic activity of clofazimine and clarithromycin in an aerosol mouse model of Mycobacterium avium infection. Antimicrob. Agents Chemother. 2020, 64, e02349. [CrossRef]

33. Zhang, S.; Chen, J.; Cui, P.; Shi, W.; Zhang, W.; Zhang, Y. Identification of novel mutations associated with clofazimine resistance in Mycobacterium tuberculosis. J. Antimicrob. Chemother. 2015, 70, 2507-2510. [CrossRef] [PubMed] 